\title{
Intermédialités
}

Histoire et théorie des arts, des lettres et des techniques

Intermediality

History and Theory of the Arts, Literature and Technologies

\section{Jean Dupuy : le je(u) du traduire anagrammatique}

\section{Barbara Bourchenin}

Numéro 27, printemps 2016

traduire

translating

URI : https://id.erudit.org/iderudit/1039814ar

DOI : https://doi.org/10.7202/1039814ar

Aller au sommaire du numéro

Éditeur(s)

Revue intermédialités (Presses de l’Université de Montréal)

ISSN

1920-3136 (numérique)

Découvrir la revue

Citer cet article

Bourchenin, B. (2016). Jean Dupuy : le je(u) du traduire anagrammatique.

Intermédialités / Intermediality, (27). https://doi.org/10.7202/1039814ar
Résumé de l'article

Jean Dupuy est un artiste singulier qui, depuis les années 1960, ne cesse de redéfinir sa pratique, de défaire ses habitudes, de renouveler ses méthodes. Cette quête de réinvention le pousse, en 1973, à engager une pratique d'auto-traduction intermédiale de son oeuvre. La description anagrammatique de ses pièces implique une réflexion sur le traduire en tant que pratique plastique à part entière. Cet article se propose d'aborder les principaux enjeux de la traduction intermédiale. Sorti du régime binaire structuraliste de traduction, le traduire mis en jeu par les anagrammes de J. Dupuy relève d'un traduire comme " jeu », bricolage intempestif et " re-créatif » du mot. Artiste traducteur-plasticien, Jean Dupuy interroge également à travers le traduire la relation du spectateur à l'oeuvre. Il met en place une biopolitique de la médiation artistique, faisant de chaque oeuvre autant d'adresses au spectateur.
Ce document est protégé par la loi sur le droit d'auteur. L'utilisation des services d’Érudit (y compris la reproduction) est assujettie à sa politique d'utilisation que vous pouvez consulter en ligne.

https://apropos.erudit.org/fr/usagers/politique-dutilisation/ 


\title{
Jean Dupuy : le je(u) du traduire anagrammatique
}

\author{
BARBARA BOURCHENIN
}

La machine - On voit, à travers une vitre, dans un espace fermé $(70 \times 60 \times 60 \mathrm{~cm})$ circonscrit dans une boîte $(180 \times 60 \times 60 \mathrm{~cm})$, un latex situé en bas de l'espace (il est tendu au-dessus d'un hautparleur). En haut de cet espace, on voit la lentille ronde d'un projecteur de lumière. Un pigment rouge sang (densité 1,56) est posé sur le latex. Un observateur fait face à la vitre. On va poser le micro d'un stéthoscope électronique au niveau de son cour, et entendre, alors, ses pulsations : elles vont agir sur le latex. Celui-ci, en suivant les rythmes cardiaques, va, en quelques minutes, projeter et remplir l'espace de pigment rouge, que l'on va voir seulement dans le rai du projecteur. Il a une forme géométrique qui ressemble à une pyramide (on croit voir un cône dans la partie haute du rai : trompe-l'œil!). C'est pourquoi la machine s'appelle Cône Pyramide. Mais j'avais d'abord donné à la machine le titre de Heart beats dust (i. e. "Le cour bat la poussière »), car en battant la poussière, le son du cour amplifié électroniquement devenait le principal agent "constructeur" de la sculpture. C'était donner au cour une deuxième fonction: celle de montrer à un observateur une réflexion directe, vibrante, vivante de lui-même ${ }^{l}$.

eart beats dust. Le cour bat la poussière (voir la figure 1). Stéthoscope au creux de la poitrine, le spectateur active l'œuvre. Ce projet technologique, liant l'art et la vie, est l'un des premiers de Jean Dupuy. L'artiste répond à un appel à concours d'Experiments in Art and Technology (E.A.T.), organisme alors dirigé en partie par un ingénieur, Billy Klüver, et l'artiste Robert Rauschenberg. Leur volonté est de marier l'art et la technologie, moyennant une collaboration entre artistes et ingénieurs. Pour l'occasion, Jean Dupuy réalise donc cette machine à voir les battements de son cœur. Les années 1980

\footnotetext{
${ }^{1}$ Extrait de l'entretien entre Jean Dupuy et Éric Mangion, paru dans le catalogue de l'exposition Jean Dupuy, à la bonne heure !, Semiose gallerie (dir.), Paris, Semiose éditions, 2008, p. 13-14.
} 
marquent par ailleurs un tournant dans son travail ${ }^{2}$ : électron libre de la création contemporaine, il développe une pratique singulière de «descriptions anagrammatiques » de ses propres œuvres, anagrammes ekphrastiques aux accents tautologiques ${ }^{3}$ qui unifient sa production. Cette description, en tant que pratique plasticienne, est une manière de redéfinir le traduire non pas dans une acception linguistique ${ }^{4}$, mais dans une acception plastique. Car ici, la traduction se fait transposition d'une œuvre, d'un langage plastique à l'autre: de la sculpture technologique à l'anagramme langagière (voir la figure 2). L'hétérogénéité des médiums nous invite à penser une traduction qui prend place dans un régime intermédial. L’ekphrasis anagrammatique, «belle infidèle », ne nous permettrait-elle pas de sortir de la dichotomie classique opposant traduction fidèle et traduction libre ?

L'étude des anagrammes a une visée double : analyse d'une pratique dans un premier temps, elle est également reconsidération de la figure de l'artiste. Le traduire à l'œuvre chez Jean Dupuy, non content de redéfinir le territoire de la traduction, nous donne à penser une nouvelle figure de l'artiste, modeste, travailleur actif et prolixe, qui, l'espace d'un instant, redevient amateur. Ainsi,

\footnotetext{
2 L'artiste délaisse sa pratique de «metteur en scène » de performances : « En 1978, je m'essoufflais sans doute. [...] J'ai donc arrêté les performances. », Ibid., p. 84. «J'entrais dans un nouveau monde avec beaucoup de détermination et beaucoup d'excitation. [...] Il me fallait trouver un tout autre environnement pour me livrer entièrement et réussir le pari dans lequel je m'étais engagé - Allé ! Lulu. », Ibid., p. 86-87. La pratique anagrammatique de Dupuy est un nouveau souffle, un défi que l'artiste se lance à lui-même.

3 «Une anagramme se contient, comme la niche son chien. Une anagramme est une tautologie. L'anagramme est un équilibre parfait des matières, elle nécessite que l'on restitue ici ce que l'on a pris là, exactement, sans aucune déperdition. En fin de compte, une anagramme est un écosystème sémantique. Le sort d'une anagramme est d'être liée à sa moitié, à son "autre" inséparable et réciproque. Une anagramme est bien révélatrice d'un sens autre, en ce sens, justement, qu'elle voit double ». Christian Xatrec et Jean Dupuy, «Stratégie de l'usure. Jean Dupuy : passeur de (son) temps », conférence lors du colloque international «L'usure : excès d'usages et bénéfices de l'art », Université Bordeaux-Montaigne, décembre 2013. La captation de la conférence est disponible sur le site de RadioFrance, «Stratégie de l'usure et passeur de (son) temps par Christian Xatrec et Jean Dupuy», «Conférences», France Culture, Radio France, 27 avril 2014, http://www.franceculture.fr/conferences/bordeaux-montaigne/strategie-de-lusure-et-passeur-de-sontemps-par-christian-xatrec-et (consultation le 26 janvier 2017). Nous signalons également la parution récente, faisant en partie suite au colloque, de l'ouvrage double dirigé par Amélie de Beauffort et Pierre Baumann: L'usure: La chaleur de l'usure (vol.I), Excès d'usages et bénéfices de l'art (vol.II), Bordeaux, Presses universitaires de Bordeaux et Académie royale des Beaux-Arts de Bruxelles, 2016.

${ }^{4}$ Fabienne Durand-Bogaert, « Oublier l'image, tendre l'oreille », Nouvelle revue d'esthétique, Paris, $\mathrm{n}^{\circ} 3,2009, \mathrm{p} .27-30$. « [...] la traduction [à visée scientifique] est présentée comme le processus par lequel un texte encodé d'une certaine manière dans une langue dite "de départ", passe, encodé d'une manière autre mais ressemblante, dans une langue dite "d'arrivée" ", Ibid., p. 28. La traduction linguistique et littérale est de l'ordre du "code » et de la transposition d'un système d'unité à un autre, dans des conditions d'équivalences mathématiques.
} 
comment penser la question de l'auto-traduction intermédiale dans l'œuvre de Jean Dupuy? Traduire, produire du texte à partir d'objets, est-ce ne rien faire, mettre en suspens sa pratique ? Il s'agira « [...] de revenir sur l'emploi d'un tel procédé systématique, à partir duquel Jean Dupuy use son temps, feint l'ennui et l'art lazy, [...] et de ce qu'il enseigne pour la Recherche (du temps perdu) ${ }^{5}$ ». L'anagramme, est-ce ce temps mort dans la pratique de l'artiste ? Un temps perdu, latent, retrouvé ? Un temps(tant) pour tout $(\tan t)$ ?

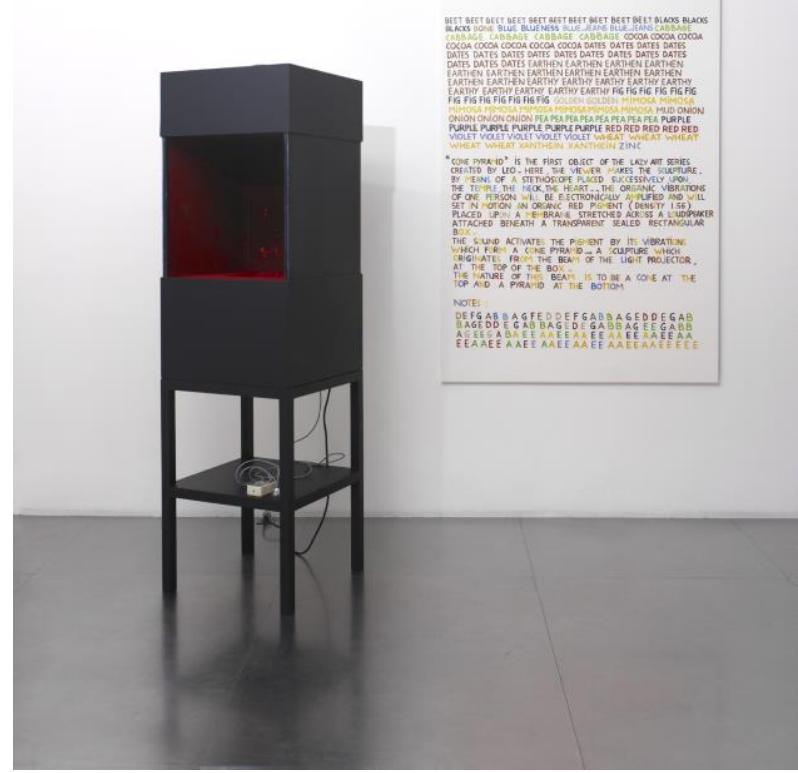

Fig. 1. Jean Dupuy, Cone pyramid (Heart beats dust), 1968, techniques mixtes, 163 x 43,5 x $48 \mathrm{~cm}$, Collection Frac Bourgogne et Jean Dupuy; Cone pyramid (anagramme), 1985, Acrylique sur toile, 184 x $133 \mathrm{~cm}$, Collection Frac Bourgogne. Photo : Fabrice Gousset. Courtesy Galerie Loevenbruck. (C) ADAGP, Paris 2016

5 Pierre Baumann, « Jean Dupuy : anagramme et ironie, le langage démonté / l'apanage du moins J., ami monnayeur de lettre engagé », dans José Quaresma, Alys Longley, Fernando Rosa Dias (dir.), Recherche en art, ironie, critique et assimilation des méthodes, Lisbonne, Centro de Filosofia da Faculdade de Letras da Universidade de Lisboa, 2015, p. 185. 
BEET BEET BEET BEET BEET BEET BEET BEET BEET BEET BLACKS BLACKS BLACKS BONE BLUE BLUENESS BLUE-JEANS BLUE_JEANS CABBAGE CABBAGE CABBAGE CABBAGE CABBAGE COCOA COCOA COCOA COCOA COCOA COCOA COCOA COCOA DATES DATES DATES DATES DATES DATES DATES DATES DATES DATES DATES DATES DATES DATES DATES DATES EARTHEN EARTHEN EARTHEN EARTHEN EARTHEN EARTHEN EARTHEN EARTHEN EARTHEN EARTHEN EARTHEN EARTHEN EARTHY EARTHY EARTHY EARTHY EARTHY EARTHY EARTHY EARTHY EARTHY EARTHY FIG FIG FIG FIG FIG FIG Fig FIG FIG Fig FIG FIG FIG GOLDEN GOLDEN MIMOSA MIMOSA MIMOSA MIMOSA MIMOSA MIMOSAMIMOSA MIMOSA MUD ONION ONION ONION ONION PEA PEA PEA PEA PEA PEA PEA PEA PURPLE PURPLE PURPLE PURPLE PURPLE PURPLE RED RED RED RED RED VIOLET VIOLET VIOLET VIOLET VIOLET WHEAT. WHEAT WHEAT WHEAT WHEAT XANTHEIN XANTHEIN ZINC

" CONE PYRAMID" is THE FIRST OBjECT OF THE LAZY ART SERIES CREATED BY LEO - HERE, THE VIEWER MAKES THE SCULPTURE BY MEANS OF A STETHOSCOPE PLACED SUCCESSIVELY UPON. THE TEMPLE, THE NECK, THE HEART - - THE ORGANIC VIBRATIONS OF ONE PERSON WILL BE ELCTRONICALLY AMPLIFIED AND WILL SET IN MOTION AN ORGANIC RED PIGMENT (DENSITY I.56) PLACED UPON A MEMBRANE STRETCHED ACROSS A LOUDSPEAKER ATTACHED BENEATH A TRANSPARENT SEALED RECTANGULAR BOX -

THE SOUND ACTIVATES THE PIGMENT BY ITS VIBRATIONS WHICH FORM A CONE PYRAMID $\rightarrow$ A SCULPTURE WHICH ORIGINATES FROM THE BEAM OF THE LIGHT PROJECTOR, AT THE TOP OF THE BOX

THE NATURE OF THIS BEAM IS TO BE A CONE AT THE TOP AND A PYRAMID AT THE BOTTOM

NOTES :

DEFGAB B A GFEDEFGABBAGEDDEGAB $B A G E D D E G A B B A G E D E G A B B A G E E G A B B$ $A G E E G A B A E E A A E E A A E E A A E E A A E E A A$ EEAAEE A AEE AAEEAAEE AAEEAAEEEEE

Fig. 2. Jean Dupuy, Cone pyramid (anagramme), 1985, acrylique sur toile, $184 \mathrm{x} 133 \mathrm{~cm}$, Collection Frac Bourgogne. Photo : Fabrice Gousset. Courtesy Galerie Loevenbruck. (C) ADAGP, Paris 2016 
Les anagrammes sont dans un premier moment un moyen de redessiner les enjeux d'une traduction intermédiale résolument post-structuraliste. Le passage d'un médium à l'autre, et l'acceptation de leur hétérogénéité respective, fait évoluer le traduire vers un acte spéculaire réflexif, conscient de lui-même. Véritable « jeu », la traduction devient cet «à-peu-près » révélateur de l'entredeux que ménage cet acte langagier. Les anagrammes, en tant que pratique heuristique du mot, n'ouvrent-elles pas une nouvelle épistémologie de l'art? L'anagramme, en tant que modèle de recherche singulier, ne permet-elle pas une redéfinition de l'acte traductif en lui-même ? Le traduire est également affaire d'agent: Jean Dupuy fait figure d'artiste-traducteur, amoureux du bon mot. Enfin, la traduction langagière en art ne cesse de rejouer des problématiques intermédiales. Favorisant ce type d'approche, c'est le visuel en tant qu'interface, le «tiers pictural $»^{6}$ qu'ont en partage installation et anagramme, qui nous permet de penser la cohabitation de deux langues au sein de cette même pratique. L'anagramme est également actrice d'une «biopolitique de la traduction ${ }^{7} »$, d'une praxis de l'art. Volonté de mise en évidence des hétérogénéités constitutives, le traduire comme théorie de la différenciation permet de repenser les modes de circulation, de diffusion et de réception des œuvres « mêlées ».

\section{LES ENJEUX DE LA TRADUCTION INTERMEDIALE DANS L'CEUVRE DE JEAN DUPUY}

\section{LA TRADUCTION ANAGRAMMATIQUE A L'ERE DU POST-STRUCTURALISME : RELATIONS TRANSESTHETIQUES ET EQUIVALENCE LINGUISTIQUE FEINTE}

Jean Dupuy jongle donc, à la manière de l'artiste-farceur qu'il est, entre plusieurs pratiques et différents médiums. Cette hétérogénéité assumée des matériaux rend nécessairement caduque la définition de la traduction telle que donnée par la mouvance structuraliste. Délaissant les hiérarchies mortifères, Jean Dupuy, bien loin également des considérations datées de l'ut pictura poesis, prône une irréductibilité de l'image au texte. Irréductibilité qui dépasse toute question de subordination du texte-traduit à son texte-source, de soumission de l'image au texte, ou inversement. Cette attitude, qui différencie

\footnotetext{
6 Liliane Louvel, Le tiers pictural : pour une critique intermédiale, Rennes, Presses Universitaires de Rennes, coll. «Interférences », 2010, p. 9.

7 Jon Solomon, «Traduction, violence et intimité hétérolinguale », dans Rada Iveković (dir.), Translating Violence, Transversal, vol. 11, n 7, 2007, www.eipcp.net/transversal/1107/solomon/fr (consultation le 23 février 2016).
} 
les systèmes, nous permet non seulement de faire évoluer la question du traduire dans un régime d'intermédialité, mais aussi de penser la traduction linguistique depuis la traduction intermédiale : en termes d'irréductibilité des textes et des œuvres ${ }^{8}$.

Quand J. Dupuy décide, de manière systématique et obsessionnelle, d'écrire une anagramme pour accompagner ses œuvres, il ne réalise pas une mais deux traductions. La première est celle qui accomplit le passage de la production «sculpturale » à la production «langagière »: l'artiste décrit son œuvre et passe ainsi d'un système visuel à un système verbal ${ }^{9}$. La deuxième traduction est celle qui se joue au sein même de l'anagramme : la dynamique anagrammatique, véritable gymnastique mécanique ${ }^{10}$, est une traduction sous forme de réagencement ${ }^{11}$. Le traduire chez Jean Dupuy est donc une traduction «à la puissance deux ${ }^{12}$ »: l'anagramme est la traduction-transposée de la traduction-description. Pour reprendre l'explication de Bernard Vouilloux, le premier passage participe d'une «relation transémiotique ${ }^{13}$ », tandis que le réagencement anagrammatique (qui fait émerger une signifiance seconde) est une « procédure de traduction ${ }^{14} »$.

La traduction, entendue en des termes linguistiques, avait, semble-t-il, vocation d'équivalence. Qu'advient-il de ce système économique en traduction

8 À propos de la différence de systèmes, de la différence fondamentale entre discours et peinture, voir Régis Debray, Vie et mort de l'image : une histoire du regard en Occident, Paris, Gallimard, coll. « Folio », 1994, p. 75-76.

9 Nous nuancerons plus tard notre propos, les anagrammes de J. Dupuy ayant un fort potentiel visuel.

10 La mécanique fait ici référence à l'œuvre Lazy Susan (Jean Dupuy, 1979), première anagramme plastique qui marque la transition entre l'art technologique et les anagrammes de l'artiste.

11 Selon le Centre national des ressources textuelles et lexicales, « l'anagramme est une interversion des lettres qui composent un mot (ou plus rarement un syntagme ou une phrase) de manière à faire un autre mot (ou un autre syntagme ou une autre phrase ». " Portail lexical : anagramme », Centre national des ressources textuelles et lexicales, 2012, www.cnrtl.fr/definition/anagramme (consultation le 14 janvier 2017). Complétons cette définition «mécanique» par celle, « sémantique », de Christian Xatrec : "Une anagramme est une opération qui consiste en une atomisation du langage et qui a pour objet la transmutation du sens. Son but n'est pas tant de générer du sens que de discerner du sens là où il est déjà, quelque part dans l'univers d'un quotidien sémantique. » Xatrec et Dupuy, 2013.

12 Myriam Suchet, «La traduction, une éthique de la ré-énonciation », Nouvelle revue d'esthétique, Paris, $n^{\circ} 3,2009$, p. 32.

13 Bernard Vouilloux, Langages de l'art et relations transesthétiques, Paris, Éditions de l’Éclat, coll. «Tiré à part», 1997, p. 16. «[Les relations transsémiotiques] seront définies comme des relations transesthétiques externes : elles recouvrent aussi bien le passage d'un art à un autre qu'un changement de médium à l'intérieur d'un art donné. »

14 Concernant la distinction entre « relations intersémiotiques » et «procédures de traduction », Ibid., p. 11. 
intermédiale ? Car, même lorsque le référent est commun (à la pièce sculpturale et à la pièce anagrammatique), les productions plastique et discursive ne sont pas concomitantes : elles ne s'équivalent pas sur le plan quantitatif, le commerce de l'une à l'autre étant faussé par une économie d'échanges boiteux. La différence matérielle est constitutive de la différence systémique: elle évacue toute possibilité de traduction de type structuraliste. Et quand bien même, au sein de l'anagramme, l'équivalence quantitative semble être respectée (un «tant pour tant » didactique de lettres employées ${ }^{15}$ ), c'est au niveau qualitatif-sémantique (au niveau de la signifiance, donc) que l'équivalence ne peut être obtenue. Le principe d'équivalence en traduction devient dès lors principe d'indifférence : «Alors, bien vite, le haut vaut le bas, le principe d'une équivalence relative met à mal tout critère de qualité [...]. L'indifférence esthétique paraît [...]. Elle feint stratégiquement l'ignorance par équivalence (tout se vaudrait). On sait bien que c'est aussi un artifice de la pensée qui opère comme un postulat nécessaire $[\ldots]^{16}$. » Chez Dupuy, feindre l'équivalence linguistique-anagrammatique est une stratégie de remise en question de toute pratique structuraliste et catégorielle d'indifférenciation et d'homogénéisation. La pratique anagrammatique révèle aussi par là même l'envers du principe d'équivalence en traduction: une équivalence feinte qui occulte les rapports de force et de résistance inhérents au traduire $^{17}$.

15 À propos des anagrammes, partagées «en deux parties parfaitement équivalentes » (Baumann, 2015, p. 194), la note de bas de page nous éclaire sur le système de différenciation entre les deux parties de l'anagramme : «Dupuy a plusieurs manières de tracer l'horizon qui partage le haut du bas de l'anagramme : en laissant un espace blanc, en traçant un trait horizontal (assez rarement), à partir d'un réseau de lignes qui relient entre elles les lettres alignées horizontalement entre le haut et le bas (pour les anagrammes qui tiennent en peu de mots). Cette dernière solution est la plus didactique. Elle illustre la vérification des correspondances. », Ibid., p. 194.

16 Ibid., p. 188.

17 «C'est qu'il est de plus en plus reconnu que la traduction n'est pas la recherche d'équivalences statiques au niveau des signes et de leurs signifiés [...], mais qu'il s'agit d'un phénomène pragmatique mettant en jeu des facteurs extralinguistiques dynamiques.» Roda P. Roberts et Maurice Pergnier, "L'équivalence en traduction», dans André Clas (dir.), Méta: journal des traducteurs, vol. 32, n ${ }^{4}$, décembre 1987, p. 392. 
LE SPECULAIRE $^{18}$, « UN PLI PAS NET » :

SORTIE DE LA LOGIQUE BINAIRE DU TRADUIRE

Ainsi, au cœur même de l'anagramme, en son «milieu» (tant géométrique qu'écologique), se joue un pli. Mais pas de n'importe quel type : il s'agirait de penser, avec Dupuy, un pli «pas net». Au niveau étymologique de l'anagramme se joue déjà la rupture, le renversement, le sens inverse et le contre-pied du nouveau ${ }^{19}$. Car, en effet, " "la figure [anagrammatique] part d'un pli du langage [...] qui l'articule dans l'ombre." Ce pli du langage n'est pas net $^{20} »$. La traduction spéculaire est ici remise en question. Très peu d'anagrammes sont partagées de manière physique : le trait horizontal présent quelquefois disparaît souvent au profit d'un espace blanc, qui mime cet effet de miroir déformant. C'est que, dans la traduction anagrammatique, le pli ne se fait pas axe de symétrie. Complication du phénomène, il est une manière de penser la complication phénoménale de la traduction linguistique : un traduire que concrétise le contact, l'effleurement non concomitant d'un haut et d'un bas induit par une pliure informelle. Ainsi, faire de l'anagramme une symétrie spéculaire selon un axe "pas net", c'est sortir de la logique binaire que le structuralisme a longtemps contribué à construire. Dans l'œuvre Sans titre (Jean Dupuy, 1993), la mise en page de «l'anagramme typographique ${ }^{21} »$ exploite directement l'effet-miroir de la ligne d'horizon que Dupuy ne trace qu'à moitié, entre partie haute et partie basse de l'anagramme : «L'anagramme est composée de deux paragraphes, il vaudrait mieux dire, le dessus et le dessous [...], pour construire une histoire continue, mais décousue (et recousue) ${ }^{22}$.» (voir la figure 3)

18 Notons que Jean Dupuy réalise des pièces qui utilisent les miroirs en tant que dispositifs renversants, qui mettent l'espace sens dessus dessous. En 1973, il organise dans son loft new-yorkais une exposition collective, «About 405 East $13^{\text {th }}$ Street \#1 », dans laquelle il réalise une pièce qui, à la manière de ses travaux anagrammatiques, met en jeu le renversement et l'illusion spéculaire : « J'ai ouvert le plancher pour voir le dessous, et le plafond, le dessus. » Dupuy, cité dans Jean Dupuy, à la bonne heure!, 2008, p. 29.

19 «Le mot anagramme est construit à partir du préfixe ana-, qui vient du grec "de bas en haut", "en arrière" ou "en sens inverse" et "de nouveau". C'est fou comme ça colle. » Baumann, 2015, p. 190.

20 Ibid., p. 186.

21 L'anagramme typographique fait ici référence aux interrogations plastiques que le «poème typographique» de Stéphane Mallarmé, Un coup de dés jamais n'abolira le hasard (1897), engageait.

22 Ibid., p. 185. 
Le pli-trait n'est pas continu, pas net donc, il déjoue le spéculaire, modélisation séculaire de la représentation mimétique et de la traduction ${ }^{23}$. L'anagramme est une «œuvre-en-valise » qui pense un spéculaire en termes d'inclusion : la pensée anagrammatique est incluse dans la pensée plasticienne générale de l'artiste. Elle met la pensée à l'envers, sur l'envers, fait voir le revers d'une vêture, le revers d'une pratique, comme on retourne sa veste: l'anagramme se fait pratique opportuniste du hasard heureux.

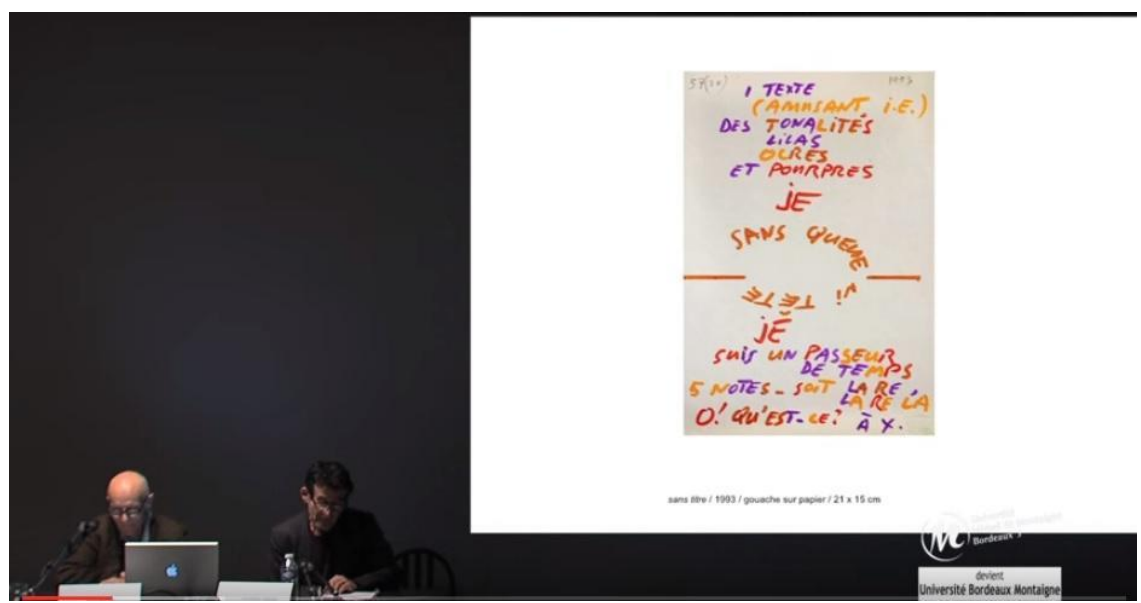

Fig. 3. Projection de Jean Dupuy, Sans Titre, 1993, gouache sur papier, 21 x $15 \mathrm{~cm}$.

Source: Christian Xatrec et Jean Dupuy, «Stratégie de l'usure. Jean Dupuy: passeur de (son) temps », conférence lors du colloque international «L'usure : excès d'usages et bénéfices de l'art », Université Bordeaux-Montaigne, décembre 2013.

Avec l'aimable autorisation de Pierre Baumann, Université Bordeaux-Montaigne.

JEU ET TEMPS RECREATIFS : BRICOLAGE HEURISTIQUE DU MOT

Ce «pli pas net» à l'œuvre dans le travail anagrammatique de Jean Dupuy est donc le moyen de faire comprendre au lecteur qu'assurément, il y a du «jeu»dans sa pratique. Un jeu qui dit «l'à-peu-près » de la traduction intermédiale, la résistance des médiums (expression matérielle de leur

23 Évoquons ici les œuvres de Markus Raetz (Ceci cela [1992-1993], en particulier) et le mythe de Narcisse (chez qui l'eau est un plan spéculaire trouble) comme symptômes de la crise de la représentation, revendiquée chez Louis Marin. De manière plus générale, la sortie du spéculaire est un symptôme de la crise de la représentation. P. Baumann parle du «caractère spéculaire (un même nombre de lettres employées deux fois) de l'anagramme » comme «la plus belle expression de l'anti-sens», Ibid., p. 189-190. Le lecteur est également invité à découvrir l'œuvre Sans Titre [la réapparition], de Jean Dupuy (2007). Dupuy, cité dans Jean Dupuy, à la bonne heure !, 2008, p. 230. 
spécificité) que ce type de traduction implique. «Donner du jeu, c'est ouvrir du sens ${ }^{24} »$, écrit Jean-François Dusigne. Mais le « jeu » n'est pas qu'une pratique bancale : c'est avant tout une pratique heuristique, enfance de l'art, qui ouvre à une épistémologie nouvelle ${ }^{25}$. La traduction qui a du jeu est donc à la fois celle qui prend le temps de douter de son efficacité ${ }^{26}$ et, par là même, celle qui prend le temps de se retrouver. Mais tant de détours dans la pratique de l'artiste ne sont-ils pas perte de temps? Il s'agit de l'une des questions centrales de cette étude: l'exercice anagrammatique auquel se livre l'artiste est-il une mise en suspens de sa pratique ? Compte tenu de l'ampleur que les anagrammes ont prise chez Jean Dupuy, la réponse est assurément non. Pourquoi dès lors se poser la question ? N'y a-t-il pas, dans ce genre de considération, confusion entre l'écrire comme théorie et l'écrire comme pratique ? C'est l'hypothèse que nous posons ici. Cependant, cette confusion est entretenue par le statut particulier de l'anagramme chez Dupuy : un «faire [qui] théorise le fait ${ }^{27}$ », une fabrique de l'art qui porte un regard réflexif sur ce qu'elle est. Jean Dupuy est «passeur de (son) temps ${ }^{28} »$, il cherche en trouvant, il «[...] négocie un modèle de pensée, qui fonde l'essence même de la recherche: la mise en doute $[\ldots]^{29}$ ». L'anagramme érige comme principe moteur l'incertitude de toute traduction, production verbale réflexive à valeur de pratique plastique. Cette dimension réflexive est présente dans beaucoup d'anagrammes :

\footnotetext{
24 Jean-François Dusigne, « Jouer, étranger à sa propre langue », Nouvelle revue d'esthétique, Paris, $\mathrm{n}^{\circ} 3,2009$, p. 68.

25 Baumann évoque cette volonté, à travers l'étude des pratiques artistiques et leur promotion, de « concevoir des modèles de recherche » : «la frontière entre portée épistémologique de la recherche en art et système de création est poreuse ». Baumann, 2015, p. 183.

26 L'étude de l'ironie chez Baumann met en avant cette question du doute. Car celui qui ironise n'est-il pas celui qui, en feignant de savoir, révèle un peu de ce qu'il ne sait pas ? «En laissant entendre qu'on sait en disant qu'on ne sait pas, on sait aussi qu'on ne sait pas tout à fait, qu'on ne sait pas assez, pas assez précisément, pas assez bien, pas assez loin. L'ironie met en péril sa propre prétention à la connaissance. L'ironie met le penseur (chercheur) du côté de la pensée nomade. » Ibid., p. 186.

27 Ibid., p. 184.

28 Le travail de l'artiste y est envisagé comme un «passe-temps », l'artiste comme un «passeur d'une rive à l'autre, d'une frontière à l'autre; sa marchandise clandestine, c'est son temps ». Xatrec et Dupuy, 2013.

${ }^{29}$ Baumann, 2015, p. 184.
} 
$57(2 x)$

I TEXTE

(AMUSANT I.E)

DES TONALITÉS

LILAS

OCRES ET POURPRES

JE

SANS QUEUE

NI TÊTE [écrit en miroir, la tête en bas dans l'anagramme originale]

JE

SUIS UN PASSEUR

DE TEMPS

5 NOTES - SOIT LA RE

LA RE LA

O ! QU'EST-CE ? A X. ${ }^{30}$

Ici, Jean Dupuy se revendique bien passeur de temps, disposant de (son) temps pour se livrer au jeu fertile de l'anagramme. "Que la traduction soit "recréation" est admis, mais qu'elle soit affaire de "singulière originalité" (Blanchot) n'est guère concevable ${ }^{31}$.» La traduction, non plus considérée comme subordonnée à un faire plus créatif, s'émancipe. De la «récréation " à la «re-création ", il n'y a qu'un pas chez J. Dupuy. Traduire n'est donc pas une suspension de sa pratique, mais un renouvellement créatif. L'activité de traduction est très justement définie par Fabienne Durand-Bogaert en tant "qu'atelier" de la pensée : lieu-faire de tests, de bricolages successifs, lieu de l'amateur. Dans l'atelier, c'est la violence de la traduction ${ }^{32}$ que le bricolage transcende. Le choc mécanistique du traduire anagrammatique devient une opération plastique nécessaire de la lettre et des choses ${ }^{33}$.

30 Jean Dupuy, Sans titre, 1993, gouache sur papier, $21 \times 15 \mathrm{~cm}$. Notons que la pratique des anagrammes, si elle a dans un premier temps été impulsée par des œuvres antérieures, s'autonomise de toute pratique annexe et ne participe plus toujours d'un double mouvement de traduction.

31 Durand-Bogaert, 2009, p. 27.

32 «L'opération de traduction en elle-même [...] n'est jamais absolue et [...] comporte toujours une sorte de violence métaphorique envers l'énoncé ou le texte original. » Jon Solomon, 2007.

33 À ce sujet, voir la très pertinente analyse de Lazy Susan par Pierre Baumann : « [...] ensemble de mots [...] désolidarisés puis remontés dans un autre ordre avec une clarté, une évidence et une fantaisie médicale [...]. Monter, c'est faire voir ». Lazy Susan est une œuvre charnière qui marque le début de la pratique anagrammatique de Dupuy. Il engage cette pratique langagière avec une anagramme plastique. "Le mode opératoire pour fabriquer Lazy Susan et le montage 
La traduction intermédiale redéfinit donc les enjeux du traduire: non plus des enjeux uniquement linguistiques, mais aussi des enjeux plastiques $^{34}$. La traduction n'est plus dans une logique économique ${ }^{35}$, mais dans une logique heuristique de renouveau méthodologique pour une épistémologie ludique de l'art.

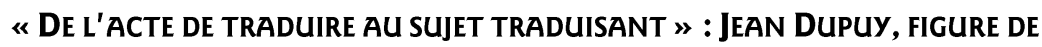 L'ARTISTE EN TRADUCTEUR}

\section{DUPUY / YPUDU : TRAVESTISSEMENT DUCHAMPIEN ${ }^{36}$ ET ETHOS DU TRADUCTEUR}

La pratique anagrammatique de Jean Dupuy est donc un faire qui non seulement réactive la créativité de l'artiste, lui apprend comment « employer son temps », mais aussi nous donne à penser la figure de l'artiste en traducteur. « De l'acte de traduire au sujet traduisant ${ }^{37} »$, il n'y a là qu'un pas. L'anagramme Sans titre, précédemment analysée, relève de ce glissement de considération de la traduction (en tant que «je[u] sans queue ni tête ») à l'agent principal de la traduction : le traducteur, le «je sans queue ni tête» dont l'identité oscille et chancelle. Cette crise identitaire ${ }^{38}$, ce questionnement sur le statut du traducteur est primordial dans la compréhension de ce qu'est le traduire. L'anagramme, si elle interroge la pratique de Jean Dupuy, n'en redéfinit pas moins l'identité de

anagrammatique se ressemblent. Autant dire que Lazy Susan est une anagramme plastique. » Baumann, 2015, p. 193.

34 Si la traduction anagrammatique permet d'ouvrir à des enjeux plastiques, elle permet également de repenser les enjeux linguistiques eux-mêmes. De la même manière, si la figure de l'artiste est positivement affectée par l'image du «traducteur », il va sans dire que la figure du traducteur tire également avantage de ce rapprochement doublement fertile.

35 Baumann veut «poser les bases de ce qu['il] nommerai[t] non pas une économie, mais une écologie de la lettre et, par extension, de ce qu'elle emporte avec elle : la créativité ». Ibid., p. 197.

36 La référence à Marcel Duchamp n'est pas anodine. Elle renvoie à son travestissement en Rrose Sélavy. Sylvie Couderc, dans un article intitulé «Portrait de l'artiste en interprète », fait même de Duchamp la figure paradigmatique de l' « artiste-interprète » (interprète au sens d'acteur, mais aussi, comme chez Dupuy, de machine à traduire l'art). Sylvie Couderc, «Portrait de l'artiste en interprète », Nouvelle revue d'esthétique, Paris, $\mathrm{n}^{\circ} 3,2009, \mathrm{p} .9$.

37 Judith Woodsworth, Horizon de la recherche de l'École doctorale Bordeaux-Montaigne, De l'acte de traduire au sujet traduisant : élaboration d'une historiographie de la traduction, Pessac (France), Maison des sciences de l'homme d'Aquitaine, Université Bordeaux-Montaigne, 24 novembre 2015.

38 À noter que nous ne pouvons parler de « crise identitaire » qu'en référence à un certain paradigme de l'identité : identité fixe et homogène au sein de laquelle la traduction viendrait faire disruption pour révéler toute l'hétérogénéité du sujet. 
son auteur. Ainsi, dans YPUDU, ANAGRAMMISTE ${ }^{39}$, l'artiste devient «Ypudu », personnage (anagrammatique lui aussi) qui s'emploie à décrire le «système » de cette gymnastique, véritable «hygiène de vie qui passe par la logorrhée de la lettre ${ }^{40} »$. Roman au genre indécidable ${ }^{41}$, livre d'artiste à l'origine d'une longue série, YPUDU, ANAGRAMMISTE met en jeu le dédoublement de la personnalité de son auteur, du narrateur et de ses personnages :

J'ai mis presque trois ans pour écrire (Hand Made!) les 85000 caractères que représentent ces quelque cent quatre-vingt-neuf pages. C'est un portrait de Léon (Léo, en anglais), qui commence à son adolescence. Il parle de sa famille, de ses amis; il décrit des objets souvent curieux qu'il crée; il invente aussi un personnage, un black bilingue et qui bégaie. Le bégaiement est un des systèmes qu'il utilise pour résoudre les équations de lettres (les anagrammes) que constituent ses textes ${ }^{42}$.

Jean Dupuy se fait artiste-écrivain ${ }^{43}$, sous le titre «YPUDU, ANAGRAMMISTE », mettant en scène ${ }^{44}$ Léon (devenu par le détour de la traduction «Léo ») qui lui-même invente un personnage bilingue qui de surcroît bégaie, dédoublant à l'infini sa propre parole. Jean Dupuy est donc tout cela à la fois: homme bégayant, féru de bi-linguismes, inventeur de personnages et conteur d'histoires à la percussion douce. Ces personnages enchâssés en disent long sur la personnalité bigarrée de cet artiste aux mille facettes.

«L'éthos » d'YPUDU, traducteur anagrammatique, transcende la figure de Jean Dupuy : «L'ethos désigne [...] la manière dont l'énonciateur construit une image de lui-même sur le plan de l'énonciation et non ce qu'il affirme de lui-même sur le plan des énoncés. Autrement dit, l'ethos concerne l'inscription

\footnotetext{
39 Jean Dupuy, Ypudu, Anagrammiste, New York, Christian Xatrec publication, 1987.

40 Baumann, 2015, p 191.

41 «Roman formel ou mode d'emploi des anagrammes? Le double perdure-t-il encore aujourd'hui ? YPUDU, ANAGRAMMISTE. Si c'est un roman, il est picaresque, et encore, je ne pense pas que ce soit un roman. Formel ? Certes, son écriture est basée sur l'anagramme. » Dupuy, cité dans Jean Dupuy, à la bonne heure !, 2008, p. 89.

42 Ibid.

43 À propos des livres d'artistes anagrammatiques qu'il publie (et de YPUDU, ANAGRAMMISTE en particulier) : «C'est une œuvre, comme une peinture ou une sculpture, que j'ai écrite dans un bureau exigu, assis à une table, à côté d'une fenêtre, à l'Est. » Ibid., p. 90. La posture créatrice, à une table, questionne la position traditionnelle de l'artiste qui se mue, se métamorphose, par l'acte de traduction, en un écrivain. Un artiste-écrivant qui passe de l'atelier à la table : motif de dissection chirurgicale tout autant que de dîners gargantuesques.

${ }^{44}$ L'artiste a déjà mis en scène et « orchestré » de multiples performances à New York.
} 
de la subjectivité dans le langage ${ }^{45}$. » À travers ses anagrammes, Jean Dupuy se cherche à haute voix. Et dans ce jeu de travestissement, c'est la «valeur heuristique de l'éthos » (valeur de praxis traductologique) qui nous permet de penser Dupuy sous la figure de l'amateur-traductologue, agent de ré-énonciation qui « échapp[e] à la conception essentialiste de l'identité ${ }^{46}$ ». Christian Xatrec mène une analyse très pertinente de l'utilisation des $i$ dans l'œuvre Quoi (Jean Dupuy, 1984, voir la figure 4) : «Mais il y a plus. La lettre $i$, cristallisée dans son galet, sous son point dans le bois, peut être lue ou doit être lue en anglais (c'est ça la complexité aussi de certaines des compositions de Jean, c'est qu'elles sont bilingues, entre le français et l'anglais), comme "i”" I. I, je, moi. Aussi "eye", l'œil. Je est donc celui qui voit ici, mais encore une fois qui voit quoi ?: quoi ? Moi? Moi ? Quoi $?^{47} \gg$ Cette œuvre ouvre un questionnement : quoi ? Le mot est inscrit en noir, en lettres capitales, sur une planche de bois; le $i$ est un trait naturel sédimentaire, ready-made d'un art paresseux, produit par le galet. [...] Cette "récolte" opportuniste de galets, sur lesquels s'inscrivent naturellement des lettres [...], ouvre la voie à un questionnement sur la quête : à la recherche de quoi l'artiste part-il ? D'un nouvel alphabet, de mots dispersés puis retrouvés, d'une identité à rassembler.

\section{JeAn DUPUy : AMOUREUX DE LA LANGUE, AMATEUR DE BONS MOTS}

Si la figure de Jean Dupuy se lit en filigrane de ses œuvres, c'est que ses anagrammes, jeux d'esprit et de mots, font corps avec leur auteur, dans une relation amoureuse où se mêlent pulsion scopique ${ }^{48}$ et «pulsion de traduire $^{49}$ ». Faire de Dupuy un amoureux de la langue, un amateur du mot, permet de penser le traduire à travers une érotique de la traduction. Cette dimension érotique, sensuelle de la pratique anagrammatique, peut paraître surprenante. Pour comprendre la passion à l'œuvre ici, il ne faut pas remonter bien loin : la genèse de la première anagramme de Dupuy suffit à nous éclairer :

73. C'est vrai qu'à l'époque les crayons furent pour moi une source d'inspiration [voir ses œuvres (sans titre) en 1972 — crayon et collage sur papier, et Think and

\footnotetext{
45 Myriam Suchet, «La traduction, une éthique de la ré-énonciation », Nouvelle revue d'esthétique, Paris, $\mathrm{n}^{\circ} 3,2009$, p. 33.

46 Ibid.

47 Xatrec et Dupuy, 2013.

48 Jean Dupuy cultive un intérêt tout particulier pour l'optique et les mécanismes du voir.

49 Antoine Berman, L'épreuve de l'étranger, Paris, Gallimard, 1984, p. 21.
} 
Suggest en 1974]. Je suis tombé sur un crayon, par hasard, un jour de désœuvrement, sur lequel étaient marqués ces quatre mots : "American Venus Unique Red ». Pour m'occuper, j'ai essayé de faire une anagramme avec les vingt-deux lettres de ces quatre mots. C'est par tâtonnements, mais en plusieurs heures quand même, que j'ai fini par tomber sur ces quatre autres mots: « Univers Ardu en Mécanique ». Cette métamorphose m’a vraiment frappé, mais je ne me doutais pas qu'elle allait changer ma vie. Bigre! J'avais trouvé une mine inépuisable que je continue aujourd'hui encore à creuser avec autant de surprise que de plaisir. IL A L'AIR AMATEUR DE SURPRISES / AMATEUR DE PLAISIR, IL RASSURE $(25 \times 2)^{50}$.

Cette première anagramme est donc issue de travaux sur les crayons de papier ${ }^{51}$ : outils de l'écrivain, du dessinateur, ils se font matière première d'une sculpture matérielle (une action directe, sur les crayons coupés en deux dans la hauteur découverte de leur milieu) et langagière (les anagrammes). Cette pratique anagrammatique, de par sa parenté généalogique avec les crayons - outils primaires de la pensée en tout genre, devient pratique plastique à part entière. Comme le dit si bien P. Baumann, «ça raconte ce qu'il vient de se produire ${ }^{52}$ » : l'anagramme devenue Vénus d'une érotique langagière mécanique ${ }^{53}$. Il n'en fallait pas plus pour que chaque anagramme, de manière plus ou moins explicite, use et abuse de son érotique (voir la figure 5): «Quelle ellipse ! Trou/Verge... L'amour fou, l'amour vénal, l'amour ronron, le fiasco, la performance érotique... Tout y est ! [...] Vertige des mots ! Vertige de l'amour ! [...] Ébats des lettres, positions infinies ${ }^{54} »$. Les anagrammes sont en effet «[...] la suggestion des images sensitives qui naissaient à leur oreille selon que, pour citer Barthes, "ça granule, ça grésille, ça caresse, ça râpe, ça coupe : ça jouit" ${ }^{2}$ ». Anagramme : jouissance des mots et de leurs agencements.

\footnotetext{
50 Dupuy, cité dans Jean Dupuy, à la bonne heure !, 2008, p. 82-83.

51 Jean Dupuy, [Sans titre], 1972, crayon et collage sur papier; Jean Dupuy, Think and Suggest, 1974; ou encore Jean Dupuy, Lazy art : Pencil, 1974, crayon et collage sur papier, 26 x 35 cm.

52 Baumann, 2015, p. 191.
}

53 À propos de la mécanique et de la machine, l'anagramme a beaucoup en partage avec les machines célibataires de Marcel Duchamp. Par manque de temps, nous renvoyons à l'analyse de Christian Xatrec: «Une peinture anagrammatique est une machine célibataire. Les machines célibataires sont une catégorie de productions artistiques proche d'un genre. [...] "Toute machine célibataire est un système d'images composé de deux ensembles égaux et équivalents. Un ensemble sexuel et un ensemble mécanique. Elle apparaît d'abord comme une machine impossible, inutile, incompréhensible, délirante. [...]" ». La machine célibataire est une «structure iconique composée de deux modèles antagonistes désirants et mécaniques produisant des quantités intensives de plaisirs auto-érotiques. On pourrait aujourd'hui remplacer le terme de "mécanique" par celui de "sémantique" ». Xatrec et Dupuy, 2013.

54 À propos de l'anagramme Trou/Verge (Jean Dupuy, 1981), lire Arnaud Labelle-Rojoux, « Who’s that guy ?* (*C'est qui ce type ?) », cité dans Jean Dupuy, à la bonne heure !, 2008, p. 108.

55 Dusigne, 2009, p. 69. 
Jean Dupuy est donc cet amateur-traducteur qui s'abandonne à une pulsion de traduire comme à une pulsion de jeu enfantine. Étymologiquement, l'amateur dit bien la figure de «celui qui aime». Dans cette entreprise d'infidélité que sont les anagrammes (infidélité aux œuvres et infidélité inhérente), l'amateur devient amant, faisant de «fidèles infidélités » à son œuvre. L'oxymoron dit bien le paradoxe d'un amour transgressif du traduire. D'autre part, toujours dans une perspective de redéfinition heuristique de sa pratique, le traduire chez Jean Dupuy est synonyme d'abandon :

Entre la chimère de la traduction parfaite et l'éloge de la trahison, il conviendrait de faire la place à une pédagogie de l'abandon. Par là, nous entendons la mise en dialogue préliminaire entre la perte d'autorité de l'original (sur lui-même) et sa saisie en traduction (du point de vue de l'autre langue). C'est précisément à cette "croisée" [...] que se tient le plaisir (éventuellement nostalgique) de la perte. [...] Pour le dire autrement, l'abandon n'est pas nécessairement tragique : il s'obtient à force de caresses et de précautions. L'érotique du traduire est l'épreuve - la pédagogie et la pragmatique, si l'on préfère — de cet abandon ${ }^{56}$.

L'art de l'abandon, Dupuy y a soustrait de manière plus radicale avant de partir à New York, détruisant volontairement toutes ses œuvres picturales ${ }^{57}$. Ici, l'abandon est lascif, lazy, il est le principe d'une entreprise qui s'est construite avec le temps.

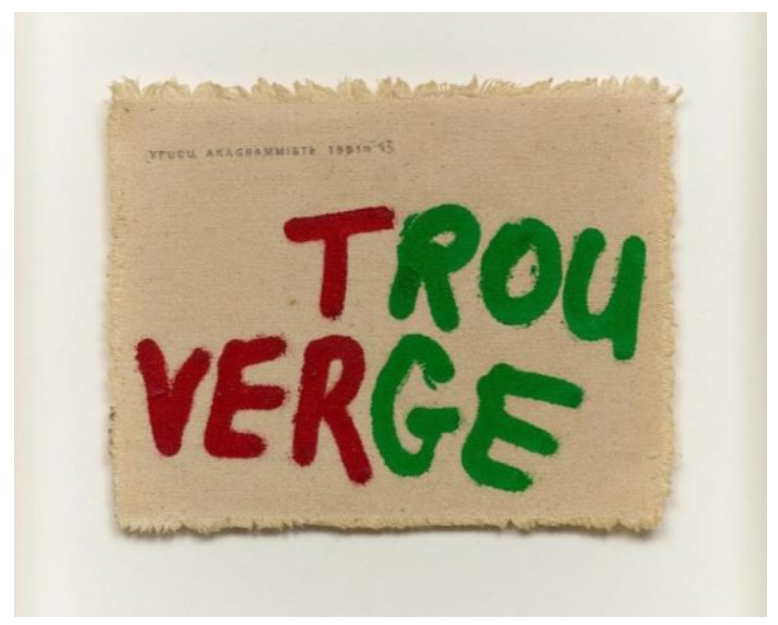

Fig. 4. Jean Dupuy, Trou Verge, 1983, gouache polychrome, mine de plomb sur tissu effiloché usagé, 15 x $20 \mathrm{~cm}$. Avec l'aimable autorisation de Drouot-Estimations. Photo : François Fernandez.

56 Sathya Rao, «Peut-on envisager l'avenir de la traduction sans plaisir ? Pour une érotique du traduire », dans André Clas (dir.), Méta : journal des traducteurs, vol. 50, n 4, décembre 2005, http://www.erudit.org/livre/meta/2005/000222co.pdf, p.5 (consulté le 28 janvier 2017).

57 Voir l'anagramme Plouf/Ouf (Dupuy, 1945). Cette anagramme décrit la destruction de ses peintures par l'artiste. 
«ÉTRANGER » SA PRATIQUE :

ENTRE LANGUE MATERNELLE ET HETEROLINGUISMES

Devenant YPUDU, Jean Dupuy se met littéralement «en traduction ${ }^{58}$ ». Ce travestissement aux accents duchampiens corrobore l'idée qu'il y a une recherche de «l'autre en soi » dans la pratique d'auto-traduction intermédiale de l'artiste: recherche d'un autre médium, d'un autre sens à l'intérieur de l'anagramme, d'un autre soi méthodologique au cœur de sa propre pratique. Il y a donc dans l'auto-traduction intermédiale ypudienne un «étrangement » à l'œuvre. Jean Dupuy, dans un même mouvement, «étrange» sa pratique et «s'étrange » à lui-même, «toute proportion gardée »: «"Toute proportion gardée", voilà [...] la vérité inthéorisable de la traduction, dont l'authenticité consiste justement en son infidélité à l'original. [Jean Dupuy] prend sur [lui] le poids de l'écart, ce que Stephen Wright a appelé le coefficient d'étrangetée ${ }^{59}$ ». L'anagramme, et la traduction à la puissance deux qu'elle engage, est également porteuse de ce «coefficient d'étrangeté », héritage direct du lien filial qui l'unit à son créateur. Antoine Berman, cité par Rada Iveković, se questionne sur la «parenté des langues ». Notons ici que Jean Dupuy s'exile volontairement aux États-Unis en 1967. Ses anagrammes (ainsi que ses interventions) négocient donc entre français et anglais, entre langue maternelle et langue du déracinement :

Par l'enracinement, l'œuvre s'enfouit dans l'épaisseur de la langue natale; par l'écart, elle s'arrache à elle en produisant une " autre langue », étrangère dès lors à la langue commune. Enracinée, l'œuvre est intraduisible. [...] Et ici advient une curieuse dialectique. [...] [La traduction] a pour «fin» de déporter l'œuvre toujours plus loin de sa langue. Mais plus une œuvre est traduite, plus s'accroît pour elle la possibilité de s'enraciner dans sa langue en apparaissant comme intraduisible. Cela n'apparaît guère au moment de sa «naissance », où ce périple n'a pas encore été effectué. Elle n'apparaît comme œuvre-de-la-langue-natale que

\footnotetext{
58 « [...] il n’y a pas non plus de degré zéro de la traduction. La langue n'est-elle pas de quelque manière toujours déjà traduction ? Le traducteur et, historiquement plus souvent, la traductrice, se met lui-même/elle-même en traduction. Elle négocie l'accueil de l'autre dans la langue d'arrivée, tout en s'y investissant personnellement »; «Stephen Wright nous dévoile le passage des frontières par la "traduction", aussi bien la traduction linguistique que cet art de vivre qu'est la mise en traduction (se mettre en traduction, se mettre à disposition) et qui fait ressortir le coefficient d'étrangeté nécessaire au truchement en tant qu'il n'est pas simplement une technique de transfert d'un terme à un autre (d'une langue, d'une œuvre...), mais dans la mesure où le passage, la translation, fait elle-même l'œuvre. » Rada Iveković, «Que veut dire traduire ? Les enjeux sociaux et culturels de la traduction », Asylon(s), n ${ }^{\circ}$ 7, 2009-2010, www.reseau-terra.eu/article889.html (consultation le 23 février 2016).
}

59 Ibid. 
lorsqu'elle est (re)traduite. On peut donc dire que la traduction accomplit le rapport de l'œuvre à sa langue ${ }^{60}$.

Si les anagrammes déterritorialisent une pratique et une langue, si elles conjurent l'enracinement mortifère d'une pratique, ce n'est que pour mieux affirmer leur parenté avec les performances, œuvres technologiques et autres productions de Jean Dupuy : «Il n'y a plus de partage, mais un geste continu, une attitude entre le langage de la forme et le langage du texte ${ }^{61}$. $\gg$ L'anagramme s'est donc faite traduction nécessaire à la révélation d'une œuvre protéiforme mais unifiée, à l'image d'un homme dont le destin s'est écrit entre deux continents et dont la trajectoire artistique s'est dessinée trans-atlantique. L'anagramme est une « reconversion franco-américaine ${ }^{62} »$.

Par ailleurs, les intrusions de la langue anglaise dans les textes de Dupuy ainsi que les systèmes d'utilisation des lettres restantes ${ }^{63}$ peuvent être analysés en tant qu' « hétérolinguismes ${ }^{64}$ ». Or, pour Myriam Suchet, « l'hétérolinguisme constitue une stratégie de relation à l'altérité65 ». Ces hétérolinguismes ne subissent pas une traduction linguistique (puisqu'ils restent in-traduits au sens strict dans les anagrammes), mais bien plutôt une traduction anagrammatique dont le propre serait de rendre visible le travail de ré-énonciation du mot : les systèmes bégayants, les notes musicales, la répétition de lettres identiques ou encore l'énumération de mots brefs et juxtaposés sont autant de marqueurs formels de la transposition anagrammatique. Cet «"appareil formel de réénonciation traductionnelle" » permet non seulement de «démythifier l'invisibilité du traducteur », mais également de penser l'anagramme comme discours : énoncé une première fois puis traduit à la puissance anagrammatique.

\footnotetext{
60 Ibid.

61 Baumann, 2015, p. 193.

62 Ibid., p. 191. L'anagramme est ainsi à l'image de son créateur. Arnaud Labelle-Rojoux interroge aussi l'identité de l'artiste : «Débutons ainsi le portrait de notre inconnu. Par son apparence et ce qui définirait au fond la nature de son art : son étrange, fondamentale, expression française, quoique, à l'instar du "French-born American" Marcel Duchamp, il soit probablement le moins hexagonal des artistes français par son mode de vie et sa curiosité. » Labelle-Rojoux, cité dans Dupuy, 2008, p. 106. L'auteur revisite la «french touch » (ou le «toucher français » selon Arnaud Labelle-Rojoux, Ibid, p. 107).

63 Voir les quatre systèmes de réemploi des lettres «orphelines » chez Dupuy. Baumann, 2015, p. 194.

64 «C'est dans un ouvrage paru en 1997 que Rainier Grutman propose le néologisme "hétérolinguisme" pour désigner "la présence dans un texte d'idiomes étrangers, sous quelque forme que ce soit, aussi bien que de variétés (sociales, régionales ou chronologiques) de la langue principale". » Suchet, 2009, p. 31. L’hétérolinguisme est défini comme « un phénomène strictement textuel ».

65 Ibid.
} 
La notion d'hétérolinguisme permet de comprendre la traduction anagrammatique comme ré-énonciation au sein même de la langue ypudienne : «Envisagée comme un acte de ré-énonciation à part entière, la traduction se voit attribuer un sujet d'énonciation autonome et responsable de sa propre stratégie discursive ${ }^{66}$. \ Les anagrammes sont bien la stratégie discursive à l'œuvre dans la pratique de Jean Dupuy : une pratique qui n'a de cesse de s'étranger.

\section{« TIERS PAYSAgE » ANAGRAMMATIQUE : TRADUCTION INTERMEDIALE ${ }^{67}$}

\section{INTERFACE VISUELLE DU TRADUIRE : TIERS PICTURAL ET VOYURE ANAGRAMMATIQUE}

Nous évoquions tout à l'heure «l'inthéorisable» à l'œuvre dans la traduction, ce «quelque chose » d'indécidable en reste, qui ne peut être soumis à une transposition. La première traduction chez J. Dupuy reste celle, transsémiotique, qui opère le passage de l'œuvre plastique antérieure à l'œuvre anagrammatique. Dans ce traduire, il n'y a pas de perte ${ }^{68}$, mais plutôt du commun en suspens. Dans Heart beats dust, du dispositif sculptural à l'anagramme, c'est le visuel qui participe de cette restance ${ }^{69}$ volatile : pigment rouge tourbillonnant, cône de lumière immatériel, muscle cardiaque se contractant. L'œuvre de Dupuy incarne, au sens littéral comme au figuré, «ce mouvement entre-deux dans la non-résolution du battement ${ }^{70} »$ Les cuvres

\section{Ibid.}

67 C'est Liliane Louvel qui, dans son ouvrage, s'interroge sur la «transposition intermédiale », qu'elle substitue à la «transposition intersémiotique » : «Il s'agira de faire le point sur ce rapport bien particulier entre langage et image, d'observer les modalités de ce que l'on nomme "la transposition intersémiotique", terme auquel je proposerai de substituer celui de "transposition intermédiale" [...]». Liliane Louvel, Le tiers pictural : pour une critique intermédiale, Rennes, Presses universitaires de Rennes, coll. «Interférences», 2010, p. 7. Bernard Vouilloux délaissait quant à lui le terme «intersémiotique » pour celui de «transsémiotique », qui transcende le projet comparatiste. Vouilloux, 1997, p. 10-11.

68 Évoquer le «reste» de la traduction en tant que «perte» nous inscrirait dans un régime essentialiste du traduire qui perpétue le régime binaire (source/cible) duquel nous sortions plus tôt.

69 Ce terme est directement emprunté à Bernard Vouilloux dans Bernard Vouilloux, La peinture dans le texte : XVIIIe-XXe siècles, Paris, CNRS éd., coll. «CNRS langage», 2005, p. 91.

70 Louvel, 2010, p. 278. Jean Dupuy, décrivant sa pièce, met l'accent sur sa dimension organique : «J'avais vu la lumière grâce aux mouvements des poussières dans la salle [de projection d'un cinéma] : il me fallait donc un projecteur. Pour la poussière, il fallait qu'elle soit très légère. C'est un ingénieur qui a trouvé un pigment de densité très faible : 1,56. Du Lithol Rubin. Ce qui m'a demandé du temps, c'est de trouver le mouvement pour agiter le pigment. C'est par une suite de coïncidences que, finalement, je suis tombé sur le mouvement le plus naturel: les pulsations cardiaques. Bravissimo ! D'autant plus que le Lithol Rubin est couleur rouge sang. J'avais là tout pour faire une sculpture de poussière. » Dupuy, 2008, p. 12-13. 
technologiques de l'artiste, dont Heart beats dust, invitent à décortiquer les mécanismes de l'attention et de la sensation ${ }^{71}$ : cette pièce en particulier renvoie au corps et à ses mécanismes réflexes les plus intimes. Elle traduit le passage d'un mouvement spontané du corps, inconscient, à sa révélation, sa prise de conscience individuelle et collective.

Ce commun en partage, que la traduction peine à transcrire, nous nous proposons d'en faire l'interface majeure de la traduction, le lieu où le traduire se joue. Nous dirons qu'il y a du «tiers » dans le traduire : «Acte éminemment stratégique, la traduction consiste, selon Berman, à "chercher-et-trouver le nonnormé de la langue maternelle pour y introduire la langue étrangère et son dire"72. » Quel serait donc cet «interstice », tiers partagé dans lequel la langue se glisse, interstice que la traduction exploite et dans lequel s'insinue l'altérité ? Avec Liliane Louvel, nous nous proposons d'envisager le «tiers pictural » à l'œuvre dans le traduire :

Je propose alors de nommer «tiers pictural » cet événement, cet entre-deux, tiers nécessaire pour analyser un certain type de textes à fort coefficient pictural. Je le construis sur le «tiers-instruit» de Michel Serres et sur le «troisième livre » de Derrida parlant de Jabès, et du livre qui reste en suspens quelque part, entre celui que le lecteur tient entre les mains et celui que l'auteur a voulu, un supplément, une « invention »dans tous les sens du terme, et d'abord celui de trésor ${ }^{73}$.

Dans les anagrammes, véritables «événement[s] de lecture ${ }^{74}$ », est donc présent ce tiers pictural, ce voir, «le tressaillement de ce "plus-à-jouir" de l'image lorsqu'elle s'interpose dans le texte littéraire et y fait tiers ${ }^{75} »$. L'anagramme est un texte littéraire mais avant tout plastique, qui se grossit du visuel en partage avec l'œuvre sculpturale : «La description [anagrammatique] serait le lieu d'un dire qui serait lui aussi un voir $[\ldots]^{76}$. » Pierre Baumann, concluant son analyse sur les anagrammes de J. Dupuy, évoque également ce «tiers » à l'œuvre chez l'artiste, qui fait sortir définitivement le traduire de toute logique binaire :

En d'autres termes, la répétition, ou plus précisément la redite déplacée (un texte pour un fait et un texte pour un texte), ne décrit pas un champ binaire, comme de

\footnotetext{
71 Nous pensons à Violet (Dupuy, 1973), une interrogation sur la fusion de l'odorat et du goût, ou encore à Ear (Dupuy, 1972), où le système optique en place permet au spectateur l'accès visuel à son propre système auditif.

72 Rao, 2005, p. 5.

73 Louvel, 2010, p. 259.

74 Ibid., p. 9. Parler d'événement de lecture n'est pas anodin. Les anagrammes ont vocation à être «performées », lues au cours d'événements.

75 Ibid., p. 225.

76 Ibid., p. 224.
} 
l'auto re-présentation, mais un "tiers paysage" (j'emprunte la formule à Gilles Clément, je la détourne, et on pourra aussi penser au tiers-instruit de Michel Serres). Quel est ce tiers paysage d'Ypudu ? C'est le territoire indécidable de l'art $[\ldots]^{77}$.

L'ekphrasis anagrammatique est bien le paroxysme du texte à fort coefficient pictural, hétérotopie visuelle foucaldienne que L. Louvel se propose de nommer une « voyure ${ }^{78}$ ». Entre lecture et vision, l'anagramme balance, d'un pôle à l'autre, du verbal au visuel : «Je lis un texte et voici de l'image, ou bien voici plus de texte encore ! En regardant l'image, je la textualise toujours de quelque façon, et en lisant le texte, je l'image. Ces actualisations sont innombrables $[\ldots]^{79}$. » Jean Dupuy, avec ses anagrammes, devient donc maître en l'art de la «voyure », art du voyou/voyeur articulant et démembrant les pratiques, en lequel il excelle depuis déjà longtemps ${ }^{80}$.

\section{ANAGRAMMES : UN VERBAL VISUELLEMENT SONORE}

Si l'étude du visuel dans les anagrammes de Jean Dupuy nous semblait essentielle pour la compréhension du traduire intermédial, la dimension sonore de ces anagrammes n'est pas non plus à négliger. Celles-ci sont donc ce verbal visuellement sonore dont les lettres s'organisent en une gamme de notes colorée: «Ainsi l'anagramme s'enrichit d'une petite méloppée [sic] élémentaire : La Ré Ré Ré, par exemple, et devient chantante ${ }^{81}$. » Ces notes de musique sont l'un des systèmes privilégiés par YPUDU pour utiliser les lettres restantes. Ainsi, Jean Dupuy fait entendre le visuel, il fait sonner cette restance :

\footnotetext{
77 Baumann, 2015, p. 196.

78 Louvel, 2010, p. 241.

79 Ibid., p. 243.

80 À ce sujet, voir la performance collective de 1977 organisée par Jean Dupuy et intitulée «Grommets \#4 ». Un « grommet» est un œillet, un trou rond par lequel les spectateurs pouvaient observer, dans la position inconfortable du voyeur, des performances artistiques.

81 Baumann, 2015, p. 194. À ce sujet, voir une œuvre récente, L'horloge musicale (JOA) (Jean Dupuy, 2008), mouvement d'horloge, système électronique, lecteur MP3, haut-parleurs, bois et gouache sur papier, 42,5 x 42,5 x 4,5 cm : «J'ai eu alors l'idée, en 2007, de faire, avec les cent trois notes [de Lazy Susan], une horloge musicale dont les heures se lisent sur vingt-quatre heures. La gamme anglaise est composée de sept lettres : C (Do) D (Ré) E (Mi) F (Fa) G (Sol) A (La) B (Si). Ainsi, les trois lettres/notes du mot "bed" (lit) chantées simultanément en trio donnent un accord musical. C'est donc sur cette relation notes et mots que j'ai dessiné l'horloge. À chaque seconde, on entend soit un solo, soit un duo, soit un trio, soit un quatuor. Finalement, le tout donne douze solos, huit duos, dix-huit trios, vingt-deux quatuors en soixante secondes. » Cette horloge est une «pièce qui trouve ici un aboutissement final comme un des longs processus qui [ont] traversé le temps. » Dupuy, cité dans Jean Dupuy, à la bonne heure !, 2008, p. 98.
} 
«Bref, l'anagramme est aussi l'art d'accommoder les restes $[\ldots]^{82}$ » et d'orchestrer leur libre expression. Le résiduel de l'opération anagrammatique, « tiers » de la traduction, est rendu visible. L'anagramme ménage visuellement la négociation et le gain que l'acte de traduire implique.

Fabienne Durand-Bogaert, dans son article «Oublier l'image, tendre l'oreille », reprend indirectement l'injonction de Louis Marin : «Donner à voir la voix, ce serait l'étrange entreprise, et peut-être le comble, de l'entreprise nommée représentation de peinture ${ }^{83}$. » L'auteur se propose en effet de «[...] se détourner de l'image, du figurable, et de porter son attention sur le pan souvent oublié de l'acte de traduire — l'écoute — [...], cheminement infiniment plus fructueux $^{84} \gg$. Cette piste méthodologique semble tout à fait applicable aux anagrammes de Jean Dupuy. En effet, ces dernières ne sont pas seulement destinées à être vues et lues, mais également à être dites: "Ainsi, une anagramme se lit à voix haute. Elle se déclame, oh oh, hi hi, de manière un peu exagérée, théâtralisée, border line, performée ${ }^{85}$. » La lecture du texte se fait donc par l'oreille : nous écoutons son intonation, l'accent et le rythme de la phrase. Nous sommes dans un type de lecture nouveau, la lecture du traducteur : « [...] les yeux seuls sont impuissants à entendre le tempo d'une phrase, à déceler les ruptures de rythme qui fondent les oppositions de style, à percevoir la couleur des sons produits par l'enchaînement des voyelles et des diphtongues ${ }^{86}{ }$. Dans Heart beats dust $^{87}$, la poussière-matière-couleur est un pigment visuel mis en mouvement par la pulsation cardiaque, la pulsation organique, intérieure, perçue par un stéthoscope ${ }^{88}$. Celui-ci n'est-il pas un instrument d'écoute, lié au son, et

82 Baumann, 2015, p. 194. Les restes sont aussi la « réserve» visible de la traduction : « [...] Rimbaud disait: "Je réservais la traduction". "Réservais" ! Dans le passage de la traduction, il se recrée une "continuité" à partir d'une discontinuité voulue (celle qui "réserve" justement). » Iveković, 2009-2010.

83 Louis Marin, «Aux marges de la peinture: voir la voix », dans Louis Marin, De la représentation, Paris, Gallimard-Le Seuil, coll. « Hautes études », 1994, p. 330.

84 Durand-Bogaert, 2009, p. 28.

85 Baumann, 2015, p. 196.

86 Durand-Bogaert, 2009, p. 29.

87 Lecture de l'anagramme de Cone Pyramid (Heart beats dust) performée par Jean Dupuy. Xatrec et Dupuy, 2013.

88 Jean Dupuy revient sur l'expérience inaugurale de sa pratique, expérience exploratoire, quasi scientifique, expérience d'écoute et d'attention enfantine : «J'étais attiré par son cabinet médical [celui de son grand-père] : les objets usuels, ceux d'un médecin de campagne (de montagne plus précisément), m'ont fasciné pendant mon enfance. Son cabinet était un petit musée vivant où je passais du temps tout seul, quand c'était possible. Je me pesais sur la balance, me mesurais le long de la toise et, mieux, j'écoutais mes pulsations avec le stéthoscope de Laennec. » Dupuy, cité dans Jean Dupuy, à la bonne heure !, 2008, p. 5. 
plus encore au rythme et à ses arythmies ? L'anagramme, lorsqu'on la lit, lorsqu'on la met en bouche ${ }^{89}$, prend une dimension sonore très importante : «BEET BEET BEET BEET BEET BEET». Les premières lignes de l'anagramme de Heart beats dust rythment la pulsation, miment par homophonie les battements du cœur, multiplient assonances et allitérations. Comme le souligne F. Durand-Bogaert, « lire, c'est donc lire avec l'oreille, prêter au texte son écoute afin de pouvoir en recueillir toutes les subtilités ${ }^{90} \gg$. L'écoute en traduction est une mise à l'épreuve de l'attention: attention de celui qui découvre la traduction, mais aussi attention de celui qui la met en œuvre. L'anagramme chez Dupuy fait en somme office de marqueur d'attention décuplée, augmentée et amplifiée :

Pour qu'une véritable écoute du texte puisse s'opérer, il faudrait que le comprendre veuille bien attendre, que l'acte d'interprétation se laisse différer. Alors peut-être le traducteur pourrait-il se mettre à l'écoute non seulement du rythme et de la scansion du texte, mais aussi — et la chose me semble d'égale importance - de son propre rythme intérieur. Chacun porte en soi un rythme propre que la parole et l'écriture mettent en évidence. Ce rythme est comparable à une ritournelle : familier et ronronnant ${ }^{91}$.

La description anagrammatique est bien cet acte de compréhension différée auquel l'auteur fait écho. Heart beats dust est la métaphore plastique de cette attentive écoute des rythmes : rythme du spectateur, de l'œuvre et d'un Jean Dupuy-traducteur qui, à la manière d'un Duchamp volontairement retardataire, diffère l'interprétation close et définitive de ses œuvres et de sa pratique. Les anagrammes incarnent «le plaisir d'une pensée qui respire (Jankélévitch), qui germe dans les interstices. La respiration, c'est un maître mot d'YPUDU. Heart Beat $[s]$ Dust avait ouvert la voie $[\ldots]^{92} »$.

En faisant usage de l'anagramme, cette «gymnastique linguistique » toujours recomposable et à recommencer, Jean Dupuy redéfinit l'acte de traduction comme toujours inachevé : « [...] un traducteur est à la fois un lecteur qui signe et écrit sa lecture, et un auditeur qui signe et écrit son écoute. [...] Traduire, dans ces conditions, ne saurait relever que d'un acte provisoire, toujours à reconduire ${ }^{93}$. » L'artiste pose aussi les fondements d'une pratique

89 À la manière des orateurs - Démosthène ne s'exerçait-il pas à la diction en mâchant des cailloux ? - « « [... j'ai pu constater combien la mise en bouche du texte, le passage organique à l'oralité contribue au surgissement du sens » (Dusigne, 2009, p. 69).

90 Durand-Bogaert, 2009, p. 29.

91 Ibid.

92 Baumann, 2015, p. 197.

93 Durand-Bogaert, 2009, p. 30. 
vivante, d'une pensée des œuvres, et de l'art dynamique et organique. Nous parlions tout à l'heure d'étranger sa langue. Tendre l'oreille, redécouvrir les œuvres « à l'oreille » sont l'un des moyens d'y parvenir :

Pour Roland Barthes, le rêve est précisément de "connaître une langue étrangère (étrange) et cependant ne pas la comprendre : percevoir en elle la différence, sans que cette différence soit jamais récupérée par la socialité superficielle du langage, communication ou vulgarité; connaître, réfractées positivement dans une langue nouvelle, les impossibilités de la nôtre; descendre dans l'intraduisible, en éprouver la secousse sans jamais l'amortir, [...]". En retour, concernant le travail sur sa propre langue maternelle [...], il me parait important d'adopter une attitude équivalente : débanaliser son usage, rendre la parole non ordinaire, pour en laisser vibrer ses multiples résonances, comme s'il s'agissait d'apprendre à la réentendre, comme une langue étrangère, quand bien même il s'agit de sa langue maternelle ${ }^{94}$.

C'est exactement la démarche de Jean Dupuy: il «étrange » sa langue maternelle à travers la pratique des anagrammes. Il « approche "en étranger" [sa] langue originelle ${ }^{95} »$. L'anagramme devient cette langue vernaculaire, étrange, qui différencie les mots, qui ouvre une «méta-poétique de l'ordinaire qui transcende les fondations du système sur lesquelles ell[e] repos[e] ${ }^{96} »$.

\section{ADRESSE DU TRADUIRE : PRAXIS SOCIALE DE L'ART}

La description anagrammatique, qui s'énonce et s'exprime, était pensée chez M. Suchet en tant que «discours». L'anagramme est bien cet acte d'énonciation, à la puissance deux ${ }^{97}$. Au-delà de son aspect plastique et des enjeux méthodologiques qu'elle pose, elle acquiert un rôle politique en se faisant praxis sociale ${ }^{98}$. Qu'entendre par là? L'anagramme chez Dupuy signe un engagement particulier, une responsabilité multiple: celle d'un artiste envers son œuvre et son public, celle des spectateurs envers la création. Les anagrammes de Jean Dupuy prennent donc place dans une «biopolitique de la

\footnotetext{
94 Dusigne, 2009, p. 69.

95 Ibid.

96 Xatrec et Dupuy, 2013.

97 Considérant les possibilités multiples d'agencements de l'anagramme, nous pouvons même l'envisager comme traduction à la puissance $n$.

98 Dans cet article à forte dimension historique, l'auteur s'intéresse à la place de la traduction dans la différence post-coloniale ou culturelle. La traduction prend ainsi place au cœur d'un débat politique très spécifique - qui excède notre domaine artistique : il s'agit pour l'auteur de «faire entrer sans détour la traduction dans un débat politiquement éclairé portant sur la production de relations sociales en même temps que de connaissances humanistes dans le contexte de la différence anthropologique héritée du colonialisme ». Solomon, 2007.
} 
traduction ${ }^{99}$ » étendue à une biopolitique de la médiation artistique (qui en passe nécessairement par une traduction dont la définition excède l'acception linguistique). Le traduire anagrammatique est au contraire un acte de pratique sociale qui construit les agents, sujets épistémologiques de la traduction, dans leur singularité : la traduction assume un rôle de constitution de la différence au sein même du système de création-réception de l'art.

Dans une logique post-structuraliste, «les deux aspects clés de la définition de la traduction selon Sakai sont les suivants : 1) la distinction entre deux moments séparés, l'adresse et la communication et 2) la position exceptionnelle du traducteur ${ }^{100} »$. Si nous avons déjà évoqué la place de choix dont le traducteur dispose, la question de la nature du traduire reste à interroger. Le traduire anagrammatique, en tant que ré-énonciation, se faisait discours chez M. Suchet. Mais tout discours n'est pas nécessairement communication. En effet, si la communication est l'aboutissement espéré de toute situation linguistique, il ne faut pas négliger le potentiel de «non-communication » du discours. Ainsi, l'anagramme nous permet de distinguer entre adresse et communication :

[...] l'«adresse » indique une relation sociale (entre l'émetteur et le destinataire du message) essentiellement pratique et performative par nature, et par là même indéterminée et ouverte à la négociation du signifié; la «communication » nomme la représentation imaginaire de cette relation sous la forme d'une série d'unités dénotées par des identités pronominales et un contenu informatif, c'est-àdire qui nous sommes supposés être et ce que nous sommes supposés vouloir dire ${ }^{101}$.

Ainsi, toute ekphrasis anagrammatique ne serait-elle pas adresse plutôt que communication? Interroger l'adresse, c'est se questionner sur les agents qui sont des témoins actifs ou passifs de la traduction. Les traductions anagrammatiques de Dupuy sont des «adresses » au spectateur : elles performent une relation entre l'œuvre et son récepteur qu'il faut actualiser en permanence. Cette autotraduction intermédiale n'est pas communication en ce sens qu'elle évacue tout contenu informatif stable et fixé qui présuppose une attitude normée et homogène chez le spectateur : elle met en place une «négociation du signifié » perpétuelle. J. Solomon le résume ainsi : «En soi, 1'“adresse” ne communique

99 Le terme de «biopolitique » appliqué à la traduction chez Jon Solomon est directement emprunté à Michel Foucault : «La biopolitique recouvre donc une sphère quotidienne de relations et d'actions sociales ostensiblement apolitiques (ou dépolitisés) - ce que Foucault appelle l'entrée de la vie dans l'histoire" - qui n'en est pas moins investie d'effets déterminants pour la production de sujets sociaux ». Ibid.

100 Solomon, 2007.

101 Ibid. 
rien, sinon la présence de la "communication" comme une possibilité susceptible d'être réalisée ou non en cours de traduction. L'adresse est donc un déclencheur de potentialité $[\ldots]^{102}$. » Les anagrammes, ces adresses au spectateur-lecteur, dénoncent un phénomène global de standardisation linguistique, elles incarnent le refus chez l'artiste de tout processus d'homogénéisation de la langue. Par ailleurs, il ne tient qu'au spectateur de faire de l'anagramme une communication : l'art et la vie que Jean Dupuy a toujours éprouvés comme intimement liés s'entrelacent ici de manière active, selon le bon vouloir du spectateur même. L'adresse anagrammatique est une manière de plus pour l'artiste de «s'étranger » et de trouver une «extériorité » à sa propre pratique ${ }^{103}$. C'est le régime d'adresse hétérolinguale, amené à son paroxysme par l'hétérogénéité des médiums, qui permet de sortir d'un régime homolingual mortifère : régime qui lissait le caractère singulier des pratiques et des agents. En cela, Jean Dupuy est passé maître au jeu d'adresse qu'est le traduire anagrammatique.

Ainsi, l'auto-traduction intermédiale chez Jean Dupuy met au jour « la relation complexe entre les langages ${ }^{104} »$. Interrogation propre au traduire, elle est le fondement de la recherche en art. Les anagrammes, «[...] vertiges méthodiques scrupuleusement poursuivis, que dis-je, recherchés avec acharnement, produisant, au-delà des jeux de langage, des récits brefs, des peintures, de la musique même ${ }^{105}$ ", sont l'expression vibrante d'une méthode nouvelle, « une méthode dans la méthode : la "méthode Dudu" !106 ». Les enjeux de cette méthode fondent une nouvelle pratique, une nouvelle adresse de l'art, mais aussi une nouvelle épistémologie, heuristique et organique, tenant du modus vivendi. La redéfinition du traduire s'est faite chez Jean Dupuy à travers le modèle de recherche singulier qu'est l'anagramme : «Elle est "figure de rhétorique" [...], "gymnastique chorégraphique, bref [...] le geste du corps saisi en action, et non pas contemplé au repos [...]. La figure, c'est l'amoureux au travail." Un performeur au grand cœur ${ }^{107}$. » Jean Dupuy, homme aux yeux d'enfant, est de ceux qui, lorsqu'on les interroge sur leur œuvre, répondent par un rébus :

\footnotetext{
102 Ibid.

103 Ibid. « [...] essayer de communiquer, c'est s'exposer soi-même à l'extériorité [...] ».

104 Baumann, 2015, p. 184.

105 Ibid., p. 113.

106 Labelle-Rojoux, 2008, p. 112-113.

107 Baumann, 2015, p. 186.
} 


\section{$\mathbf{A B}^{2}$ \\ DO ${ }^{108}$}

Génialité de la méthode et du ton, qui, lui, « échappe [...] à toute méthode ${ }^{109}$ ».

108 Dupuy, 2008, p. 102. Rébus qui résume sa pratique de manière géniale. Les trois traits font mine d'axe de symétrie factice : le DO n'est pas le reflet par symétrie axiale du AB, il est le résultat de la mise au carré du $\mathrm{AB}$, de la mise à la puissance deux du texte de base.

109 Jean Dupuy, « Anagrammes » [2006], dans Jean Dupuy, à la bonne heure!, 2008, p. 116. Au sujet des anagrammes de Jean Dupuy, voir également Jean Dupuy, Where, Paris, Les Presses du réel, 2013. 


\title{
Jean Dupuy : le je(u) du traduire anagrammatique
}

\author{
BARBARA BOURCHENIN, UNIVERSITE BORDEAUX-MONTAIGNE
}

\section{RÉSUMÉ}

$\mathbf{I}_{26}$ Jean Dupuy est un artiste singulier qui, depuis les années 60 , ne cesse de redéfinir sa pratique, de défaire ses habitudes, de renouveler ses méthodes. Cette quête de réinvention le pousse, en 1973, à engager une pratique d'autotraduction intermédiale de son œuvre. La description anagrammatique de ses pièces implique une réflexion sur le traduire en tant que pratique plastique à part entière. Cet article se propose d'aborder les principaux enjeux de la traduction intermédiale. Sorti du régime binaire structuraliste de traduction, le traduire mis en jeu par les anagrammes de J. Dupuy relève d'un traduire comme « jeu », bricolage intempestif et « re-créatif» du mot. Artiste traducteur-plasticien, Jean Dupuy interroge également à travers le traduire la relation du spectateur à l'œuvre. Il met en place une biopolitique de la médiation artistique, faisant de chaque œuvre autant d'adresses au spectateur.

\section{ABSTRACT}

$\mathbf{I}_{27}$ Jean Dupuy is an unique artist who has continued, since the 1960s, to redefine his practice, undo his habits, renew his methods. This quest for reinvention urged him, in 1973, to initiate a practice of an intermedial self-translation of his work. The anagrammatic description of his artworks involves thinking of translation as a visual art practice in itself, as a translate. This article aims to address the key issues of intermedial translation. Released from the structuralist binary system of translation, the translate involved in Dupuy's anagrams reports to a translate as a «game», unintended, and «re-creative » tinkering of the word. Through the translate, Jean Dupuy, as visual-translator artist, also questions the viewer's relationship to the work. He sets up a biopolitics of artistic mediation, making each work an address to the viewer.

\section{NOTE BIOGRAPHIQUE}

BARBARA BOURCHENIN est doctorante agrégée en arts (histoire, théorie, pratique) à l'Université Bordeaux Montaigne en France et conduit une thèse sous la direction de Bertrand Prévost au laboratoire de recherche MICA (Médiations, Information, Communication, Arts - École doctorale Montaigne-Humanités). Elle est aussi attachée temporaire d'enseignement et de recherche en arts 
plastiques à cette même université. Elle est l'auteure de "L'écrire avec de 1'“essayer dire" : pour une expérience langagière de la communauté esthétique » (Revue Postures) et de «Leçon(s) de choses. Claude Simon et Lawrence Weiner: écrire l'art et la littérature. Enjeux performatifs, mémoriels et topographiques du langage » (Fabula). 